\title{
Graph-based Codes for Quantize-Map-and-Forward Relaying
}

\author{
Ayan Sengupta*, Siddhartha Brahma*, Ayfer Özgür*, Christina Fragouli* and Suhas Diggavi ${ }^{\dagger}$ \\ ${ }^{*}$ EPFL, Switzerland, ${ }^{\dagger}$ UCLA, USA
}

\begin{abstract}
We present a structured Quantize-Map-andForward (QMF) scheme for cooperative communication over wireless networks, that employs LDPC ensembles for the node operations and message-passing algorithms for decoding. We demonstrate through extensive simulation results over the fullduplex parallel relay network, that our scheme, with no transmit channel state information, offers a robust performance over fading channels and achieves the full diversity order of our network at moderate SNRs.
\end{abstract}

\section{INTRODUCTION}

Quantize-Map-and-Forward (QMF) is a recently proposed strategy that allows to approximately achieve the capacity of arbitrary wireless relay networks [2]. QMF operates on the following principles: each relay quantizes its received signal (at noise-level) and (uniformly at random) maps it onto the transmit codebook; the destination decodes the source message without decoding the transmitted codewords (or quantized values) from the relays.

We translate these principles to a practical QMF coding scheme for the full-duplex parallel relay network [1]. Our source codebook and the relay mappings employ LDPC ensembles that allow a sparse factor graph representation, while the decoder implements a message passing algorithm on a compound graph. We here focus on the parallel relay network, however our designs extend to more general configurations.

QMF is known to be not only approximately optimal, but also robust, in the sense that relay operation needs only local knowledge, while transmit channel state information (CSI) is not required at any point in the network to achieve full cooperative diversity [3], [4]. In contrast, strategies such as opportunistic routing (OR) [7] and Amplify-Forward (AF), do require transmit CSI for best path selection or beamforming ${ }^{1}$. Even a partial decode-and-forward strategy, which approximately achieves capacity for the parallel relay network, requires transmit CSI for the rates supportable in the broadcast and multiple-access sections of the network separately and does not possess the universal approximation property for arbitrary networks [2]. Moreover, it requires superposition coding, which is not trivially implementable.

Our computationally efficient strategy exhibits similar advantages over the more traditional schemes. For example, our numerical evaluations in Section IV show that for symmetric fading channels QMF is within $3 \mathrm{~dB}$ of the cut-set bound at a

\footnotetext{
${ }^{1}$ It is a common assumption that receive CSI is available since this can be obtained during reception. However in typical fading wireless channels it is not realistic to have transmit CSI since the channel could have significantly changed by the time this information is fed back.
}

frame error rate/outage of $10^{-3}$, using only receive CSI. This performance improves when we consider asymmetric fading channels. Moreover, our scheme achieves the full diversity order of the network without transmit CSI (at moderate SNRs) and offers a cooperation gain over OR for asymmetric fading.

Main contributions: (i) We use LDPC ensembles for the encoding and relay maps, to develop a compound graphical representation of the network operation that takes into account the noise, the multiple access and the broadcast due to the wireless channels as well as the processing at various nodes such as encoding, quantization, modulation and mapping. We devise an efficient scheduling and message passing algorithm on this graph. (ii) We extensively evaluate the performance of our codes, compare them to AF/OR strategies and demonstrate that our design and implementation of QMF retains several of the advantages over them.

Related Work: Lattice codes for QMF were studied in [6]. These allow polynomial-time complexity for the relay operation, however, similar to the original construction in [2], the decoding complexity is exponential in blocklength. In [5], a QMF LDPC-based coding scheme for the half-duplex singlerelay network was presented. This scheme, to simplify the decoding problem, orthogonalizes the interfering signals by invoking a one-block delay at the relay, followed by successive interference cancelation at the destination. This approach does not maintain the desired universality property of the QMF strategy. In particular, it does not naturally extend to larger configurations since it relies on simplifying the QMF strategy, specifically for the case of a single half-duplex relay.

This paper is organized as follows: Section II details the communication model and performance metrics; Section III presents our proposed QMF-based design for encoding and decoding; and finally Section IV provides extensive numerical evaluation results.

\section{MODEL AND MetricS}

\section{A. Parallel Relay Network}

We consider the parallel relay network, where a source $S$ wants to communicate to a destination node $D$ with the help of two full-duplex relays $R_{1}$ and $R_{2}$. The signal $x_{s}$ transmitted by the source is broadcast to the relays. The received signals at $R_{1}$ and $R_{2}$ are given by

$$
\begin{aligned}
& y_{R 1}=h_{1} x_{s}+n_{R 1} \\
& y_{R 2}=h_{2} x_{s}+n_{R 2},
\end{aligned}
$$




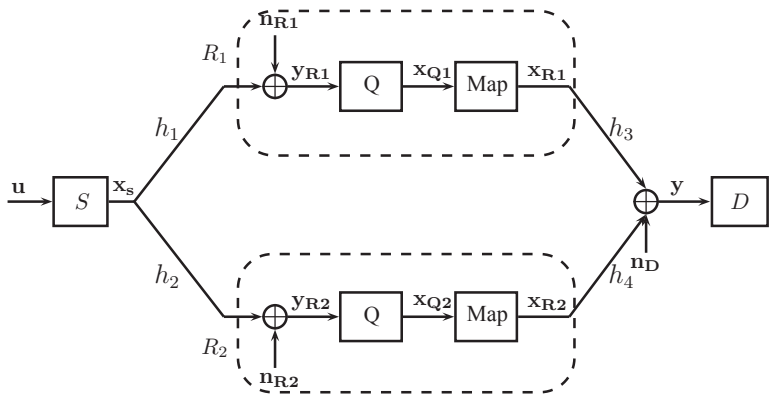

Fig. 1. QMF Strategy for the Parallel Relay Network

where $h_{1}$ and $h_{2}$ are the channel coefficients for the $S$ to- $R_{1}$ and $S$-to- $R_{2}$ channels, and $n_{R 1}$ and $n_{R 2}$ are i.i.d complex Gaussian random variables $\mathcal{C N}(0,1)$. The destination $D$ observes a superposition of the transmissions from $R_{1}$ and $R_{2}$, corrupted by its receiver noise,

$$
y=h_{3} x_{R 1}+h_{4} x_{R 2}+n_{D},
$$

where $h_{3}$ and $h_{4}$ are the channel coefficients for the $R_{1}$ to- $D$ and $R_{2}$-to- $D$ channels. The noise $n_{D}$ has the same distribution as $n_{R 1}, n_{R 2}$, and is independent of $n_{R 1}$ and $n_{R 2}$. The transmitted signals from the source and the relays are subject to an average power constraint $\mathcal{P}$.

We consider a block-fading model for the channel coefficients, where $h_{1}, h_{2}, h_{3}, h_{4}$ are independent circularly symmetric Gaussian random variables that are fixed during a transmission block.

\section{B. Traditional Cooperative Strategies}

To demonstrate the cooperation benefits of QMF and evaluate the performance of our design (detailed in section III), we compare ourselves to the following traditional cooperative schemes for the parallel relay network.

- Routing: Without transmit CSI, one of the relays is arbitrarily chosen to decode and forward the incoming messages from the source node to the destination node, while the other relay remains silent.

- Opportunistic Routing: If the magnitude of the channel realizations are available at the relays, the routing strategy could be improved to use the stronger of the two available paths. This modification of the routing strategy that employs transmit CSI is referred to as opportunistic routing.

- Amplify-and-forward: The two relays amplify their received signals to their power constraint and transmit them. The destination node effectively observes a point-to-point channel and tries to recover the transmitted message from the source. - Amplify-and-forward with Beamforming: If the phases of the channel realizations are available at the relays, the amplifyand-forward strategy could be improved by beamforming at the relays.

\section{Outage Framework}

In fading wireless channels, an important performance metric is the outage probability of the system, which is defined as the probability that the network channel realizations do not support the transmitted rate. The trade-off between (multiplexing) rate and error probability (diversity order) for wireless systems can be characterized in the high-SNR regime through the diversity-multiplexing tradeoff. In this paper we evaluate both the error performance (at a given rate) and spectral efficiency (the maximum supportable rate at given outage probability) of our QMF-based design, and compare it with the traditional cooperative schemes, in moderate SNR regimes. The robustness of QMF in fading environments has been established in [3], [4], and can be crystalized through the following simple observation.

Theorem 2.1: The QMF strategy achieves the optimal diversity-multiplexing tradeoff for arbitrary full duplex wireless networks.

The proof of this observation is immediate from the approximation result in [2].

\section{QMF SySTEM DESIGN}

\section{A. Encoding and Relaying}

Fig. 1 shows the schematic diagram of the QMF strategy for the parallel relay network. The source $S$ encodes the information vector $\mathbf{u}$ into the signal vector $\mathbf{x}_{\mathbf{s}} \in \mathcal{A}_{s}^{N}$, where $\mathcal{A}_{s}$ represents the discrete channel-alphabet at the source and $N$ denotes the transmission blocklength. The constellation $\mathcal{A}_{s}$ is constrained to have average power equal to $\mathcal{P}$, i.e., $\frac{1}{\left|\mathcal{A}_{s}\right|} \sum_{a \in \mathcal{A}_{s}}\|a\|^{2}=\mathcal{P}$

$R_{1}$ and $R_{2}$ quantize their received vectors $\mathbf{y}_{\mathbf{R} 1}$ and $\mathbf{y}_{\mathbf{R} 2}$ into $\mathbf{x}_{\mathbf{Q} 1}$ and $\mathbf{x}_{\mathbf{Q} 2}$ respectively, where $\mathbf{x}_{\mathbf{Q} 1}, \mathbf{x}_{\mathbf{Q} 2} \in\{ \pm 1\}^{b N}$, with $b$ denoting the average number of quantized bits per symbol. Subsequently, the relays map $\mathbf{x}_{\mathbf{Q} 1}$ to $\mathbf{x}_{\mathbf{R} 1} \in \mathcal{A}_{R 1}^{N}$ and $\mathbf{x}_{\mathbf{Q} 2}$ to $\mathbf{x}_{\mathbf{R} 2} \in \mathcal{A}_{R 2}^{N}$, and forward their signals to the destination. The transmit-alphabets at the relays, $\mathcal{A}_{R 1}$ and $\mathcal{A}_{R 2}$, also satisfy the same power constraint $\mathcal{P}$.

We initially focus on the binary communication problem, i.e $\mathcal{A}_{s}=\{ \pm 1\}$. We also set $\mathcal{A}_{R 1}=\mathcal{A}_{R 2}=\{ \pm 1\}$, and employ one-bit scalar quantizers at the relays. We defer the extension to non-binary constellations to section III-C.

For our source codebook $\mathcal{C}$, we use an LDPC code of the desired rate with a code-membership function $\mathbb{1}_{\left\{\mathbf{x}_{\mathbf{s}} \in \mathcal{C}\right\}}$. The theoretical development of the QMF scheme in [2] suggests that the mappings at the relays should provide sufficient and independent mixing of the incoming information streams. For the mapping at the relays, we also choose LDPC codes which induce the desirable mixing properties. The mappings can be thought of as encoding $\mathrm{x}_{\mathbf{Q}}$ with a rate $\frac{1}{2}$ code, and letting the parity bits be the transmit sequence $\mathbf{x}_{\mathbf{R}}$. In this setting the map-membership functions at the relays can be represented as $\mathbb{1}_{\left\{\left(\mathbf{x}_{\mathbf{Q} 1} \cup \mathbf{x}_{\mathrm{R} 1}\right) \in \mathcal{M}_{1}\right\}}$ and $\mathbb{1}_{\left\{\left(\mathbf{x}_{\mathbf{Q} 2} \cup \mathbf{x}_{\mathrm{R} 2}\right) \in \mathcal{M}_{2}\right\}}$, where $\mathcal{M}_{1}, \mathcal{M}_{2}$ represent the corresponding LDPC codebooks at the relays. These membership functions have a sparse factorgraph representation, which make them suitable components in iterative decoder structures.

\section{B. Iterative QMF Decoder}

We derive a compound graphical model to perform iterative decoding of the source bits $\mathbf{x}_{\mathbf{s}}$. In contrast to point-to-point decoding, the network graphical model for our iterative QMF decoder has to incorporate the following additional features: (i) 


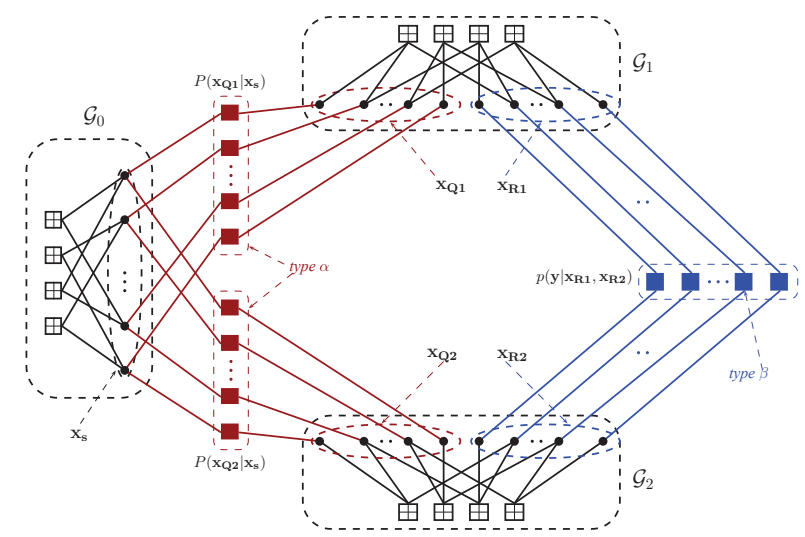

Fig. 2. Decoding Graph for binary signaling with 1-bit scalar quantizers

It should possess an explicit characterization of the functionnodes representing the quantization and multiple-access operations, together with their own set of message passing rules, and (ii) It should have a well defined informationexchange algorithm among the component LDPC Tannergraphs corresponding to $\mathcal{C}, \mathcal{M}_{1}$ and $\mathcal{M}_{2}$. In the following, we detail the derivation and key features of the graphical model.

1) Sum-Product decomposition of a posteriori probability: The decoding rule for the bit-wise MAP decoder reads:

$$
\hat{x}_{s, i}^{M A P}(\mathbf{y})=\operatorname{argmax}_{x_{s, i} \in\{ \pm 1\}} \sum_{\sim x_{s, i}} p\left(\mathbf{x}_{\mathbf{s}} \mid \mathbf{y}\right)
$$

The a posteriori probability, $p\left(\mathbf{x}_{\mathbf{s}} \mid \mathbf{y}\right)$, can be expressed as,

$$
\begin{aligned}
& p\left(\mathbf{x}_{\mathbf{s}} \mid \mathbf{y}\right)=\frac{1}{p(\mathbf{y})} \sum_{\sim \mathbf{x}_{\mathbf{s}}, \mathbf{y}} p\left(\mathbf{x}_{\mathbf{s}}, \mathbf{x}_{\mathbf{Q} 1}, \mathbf{x}_{\mathbf{Q} 2}, \mathbf{x}_{\mathbf{R} \mathbf{1}}, \mathbf{x}_{\mathbf{R} 2}, \mathbf{y}\right) \\
& p\left(\mathbf{x}_{\mathbf{S}} \mid \mathbf{y}\right) \stackrel{(a)}{\propto} \sum_{\sim \mathbf{x}_{\mathbf{s}}, \mathbf{y}} p\left(\mathbf{x}_{\mathbf{S}}\right) \cdot p\left(\mathbf{x}_{\mathbf{Q} \mathbf{1}} \mid \mathbf{x}_{\mathbf{S}}\right) \cdot p\left(\mathbf{x}_{\mathbf{Q} \mathbf{2}} \mid \mathbf{x}_{\mathbf{S}}\right) . \\
& p\left(\mathbf{x}_{\mathbf{R} 1} \mid \mathbf{x}_{\mathbf{Q} 1}\right) \cdot p\left(\mathbf{x}_{\mathbf{R 2}} \mid \mathbf{x}_{\mathbf{Q} 2}\right) \cdot p\left(\mathbf{y} \mid \mathbf{x}_{\mathbf{R} 1}, \mathbf{x}_{\mathbf{R 2}}\right) \\
& \stackrel{(b)}{\propto} \sum_{\sim \mathbf{x}_{\mathbf{s}}, \mathbf{y}} p\left(\mathbf{x}_{\mathbf{Q} \mathbf{1}} \mid \mathbf{x}_{\mathbf{S}}\right) \cdot p\left(\mathbf{x}_{\mathbf{Q} \mathbf{2}} \mid \mathbf{x}_{\mathbf{S}}\right) \cdot p\left(\mathbf{y} \mid \mathbf{x}_{\mathbf{R} \mathbf{1}}, \mathbf{x}_{\mathbf{R} 2}\right) \cdot \\
& \mathbb{1}_{\left\{\mathbf{x}_{\mathbf{s}} \in \mathcal{C}\right\}} \cdot \mathbb{1}_{\left\{\left(\mathbf{x}_{\mathbf{Q} 1} \cup \mathbf{x}_{\mathbf{R} 1}\right) \in \mathcal{M}_{1}\right\}} \cdot \mathbb{1}_{\left\{\left(\mathbf{x}_{\mathbf{Q} 2} \cup \mathbf{x}_{\mathbf{R} \mathbf{2}}\right) \in \mathcal{M}_{2}\right\}}
\end{aligned}
$$

where $(a)$ follows from the fact that $\mathrm{x}_{\mathbf{S}} \leftrightarrow\left(\mathbf{x}_{\mathrm{Q} 1}, \mathbf{x}_{\mathbf{Q} 2}\right) \leftrightarrow$ $\left(\mathrm{x}_{\mathrm{R} 1}, \mathrm{x}_{\mathrm{R} 2}\right) \leftrightarrow \mathrm{y}$ and $\mathrm{x}_{\mathrm{R} 1} \leftrightarrow \mathrm{x}_{\mathrm{Q} 1} \leftrightarrow \mathrm{x}_{\mathrm{s}} \leftrightarrow \mathrm{x}_{\mathrm{Q} 2} \leftrightarrow \mathrm{x}_{\mathrm{R} 2}$ form Markov chains, and $(b)$ follows from the uniform distribution on the source codeword, and the code and map membership constraints. Also, from the memoryless property of the channel, the terms $p\left(\mathbf{x}_{\mathbf{Q} \mathbf{1}} \mid \mathbf{x}_{\mathbf{s}}\right), p\left(\mathbf{x}_{\mathbf{Q} \mathbf{2}} \mid \mathbf{x}_{\mathbf{s}}\right)$ and $p\left(\mathbf{y} \mid \mathbf{x}_{\mathbf{R} \mathbf{1}}, \mathbf{x}_{\mathbf{R} \mathbf{2}}\right)$ further factorize on a symbol-by-symbol basis as shown in the decoder graph in Fig. 2. The decoding problem thus reduces to computing the marginal of a factorized function and choosing the value that maximizes the marginal.

2) Structure of the decoder: As shown in Fig. 2, the compound graph contains the graphs corresponding to the source codebook $\left(\mathcal{G}_{0}\right)$, and the relay maps $\left(\mathcal{G}_{1}\right.$ and $\left.\mathcal{G}_{2}\right)$ are constituents of the overall graphical structure. We observe that in addition to the variable and check nodes in $\mathcal{G}_{0}, \mathcal{G}_{1}$ and $\mathcal{G}_{2}$, two other types of function nodes enter the graph structure:

(i) The source-to-relay (type $\alpha$ ) function nodes, which connect the $\mathbf{x}_{\mathrm{s}}$ and $\mathbf{x}_{\mathbf{Q}}$ variable nodes, and represent the functions $p\left(\mathbf{x}_{\mathbf{Q} 1} \mid \mathbf{x}_{\mathbf{s}}\right)$ at $R_{1}$ and $p\left(\mathbf{x}_{\mathbf{Q} 2} \mid \mathbf{x}_{\mathbf{s}}\right)$ at $R_{2}$. These nodes correspond to the quantization operation at the relays and facilitate soft-information exchange between $\mathcal{G}_{0}$ and $\left\{\mathcal{G}_{1}, \mathcal{G}_{2}\right\}$.

(ii) The multiple-access (type $\beta$ ) function nodes, connecting the $\mathbf{x}_{\mathbf{R} 1}$ and $\mathrm{x}_{\mathbf{R} 2}$ variable nodes, and representing the function $p\left(\mathbf{y} \mid \mathbf{x}_{\mathbf{R} 1}, \mathbf{x}_{\mathbf{R} 2}\right)$. The information-exchange between $\mathcal{G}_{1}$ and $\mathcal{G}_{2}$ that occurs via these nodes is an important ingredient in harnessing the benefits of co-operation from the relays.

3) Message passing rules: The decoding proceeds via a message-passing algorithm on the decoding graph. We set all messages flowing through the edges to be in log-likelihood ratio form, i.e of the form $\ln \frac{p(x=+1)}{p(x=-1)}$. The messages passed from every variable node and also from the check nodes in $\mathcal{G}_{0}, \mathcal{G}_{1}$ and $\mathcal{G}_{2}$ follow usual belief propagation (BP) message passing rules as summarized in [8]. However, we need to illustrate the message passing rules for the type $\alpha$ and type $\beta$ function nodes that are new to the network graphical model.

Each type $\alpha$ function node $c$ is connected to a variable node $v_{0}$ in $\mathcal{G}_{0}$ and to another node $v_{i}$ in either $\mathcal{G}_{1}$ or $\mathcal{G}_{2}$. The messages passed from the type $\alpha$ function node to node $v_{0}$ in $\mathcal{G}_{0}$ and to node $v_{i}$ in $\mathcal{G}_{i},(i \in\{1,2\})$ are given by

$$
\begin{aligned}
m_{c \rightarrow v_{0}}^{\alpha \rightarrow \mathcal{G}_{0}} & =\ln \frac{p_{+1 \mid+1} \cdot p\left(v_{i}=+1\right)+p_{-1 \mid+1} \cdot p\left(v_{i}=-1\right)}{p_{+1 \mid-1} \cdot p\left(v_{i}=+1\right)+p_{-1 \mid-1} \cdot p\left(v_{i}=-1\right)} \\
& =\ln \frac{p_{+1 \mid+1} e^{m_{v_{i} \rightarrow c}^{\mathcal{G}_{i} \rightarrow \alpha}}+p_{-1 \mid+1}}{p_{+1 \mid-1} e^{m_{v_{i} \rightarrow c}^{\mathcal{G}_{i} \rightarrow \alpha}}+p_{-1 \mid-1}} \\
m_{c \rightarrow v_{i}}^{\alpha \rightarrow \mathcal{G}_{i}} & =\ln \frac{p_{+1 \mid+1} \cdot p\left(v_{0}=+1\right)+p_{+1 \mid-1} \cdot p\left(v_{0}=-1\right)}{p_{-1 \mid+1} \cdot p\left(v_{0}=+1\right)+p_{-1 \mid-1} \cdot p\left(v_{0}=-1\right)} \\
& =\ln \frac{p_{+1 \mid+1} e^{m_{v_{0} \rightarrow c}^{\mathcal{G}_{0} \rightarrow \alpha}}+p_{+1 \mid-1}}{p_{-1 \mid+1} e^{m_{v_{0} \rightarrow c}^{\mathcal{G}_{0} \rightarrow \alpha}+p_{-1 \mid-1}}}
\end{aligned}
$$

where $p_{ \pm 1 \mid \pm 1}$ denote the transition probabilities, $p\left(v_{i} \mid v_{0}\right)$, where $v_{0} \in \mathbf{x}_{\mathbf{S}}$ and $v_{i} \in \mathbf{x}_{\mathbf{Q}}$, and are obtained from the channel statistics.

Each type $\beta$ function node $c$ is connected to a variable node $v_{1}$ in $\mathcal{G}_{1}$ and to $v_{2}$ in $\mathcal{G}_{2}$. Using similar marginalizations of the corresponding functions as in the case of type $\alpha$ nodes, the messages passed from the type $\beta$ nodes are derived as

$$
\begin{aligned}
& m_{c \rightarrow v_{1}}^{\beta \rightarrow \mathcal{G}_{1}}=\ln \frac{p_{+1,+1} e^{m_{v_{2} \rightarrow c}^{\mathcal{G}_{2} \rightarrow \beta}}+p_{+1,-1}}{p_{-1,+1} e^{m_{v_{2} \rightarrow c}^{\mathcal{G}_{2} \rightarrow \beta}}+p_{-1,-1}} \\
& m_{c \rightarrow v_{2}}^{\beta \rightarrow \mathcal{G}_{2}}=\ln \frac{p_{+1,+1} e^{m_{v_{1} \rightarrow c}^{\mathcal{G}_{1} \rightarrow \beta}}+p_{-1,+1}}{p_{+1,-1} e^{m_{v_{1} \rightarrow c}^{\mathcal{G}_{1} \rightarrow \beta}}+p_{-1,-1}}
\end{aligned}
$$

where $p_{ \pm 1, \pm 1}$ represents $p\left(y \mid v_{1}, v_{2}\right)$ where $y$ is the channel observation, $v_{1} \in \mathbf{x}_{\mathbf{R} 1}$ and $v_{2} \in \mathbf{x}_{\mathbf{R} 2}$.

4) Decoding Schedule: Having defined the messagepassing rules for the variable and function nodes, it remains to specify the schedule for information exchange in the compound graphical model. From a computational standpoint, it is efficient to perform message-passing rounds within $\mathcal{G}_{1}$ and $\mathcal{G}_{2}$ in parallel, once they receive the corresponding incoming messages from the type $\alpha$ and type $\beta$ function nodes. This simultaneously updates the messages $m_{v \rightarrow c}^{\mathcal{G}_{i} \rightarrow \alpha}$ and $m_{v \rightarrow c}^{\mathcal{G}_{i} \rightarrow \beta}$ 
$(i=1,2)$. In the next step $\mathcal{G}_{0}$ performs local message-passing rounds with its inputs from the type $\alpha$ nodes, and subsequently updates $m_{v \rightarrow c}^{\mathcal{G}_{0} \rightarrow \alpha}$. This in turn updates $m_{c \rightarrow v}^{\alpha \rightarrow \mathcal{G}_{i}}$. Also $\mathcal{G}_{1}$ and $\mathcal{G}_{2}$ update their information via the type $\beta$ update equations to have new estimates for the subsequent global iteration. Such a schedule makes good use of the parallelism inherent in the network structure and is illustrated in the algorithm below.

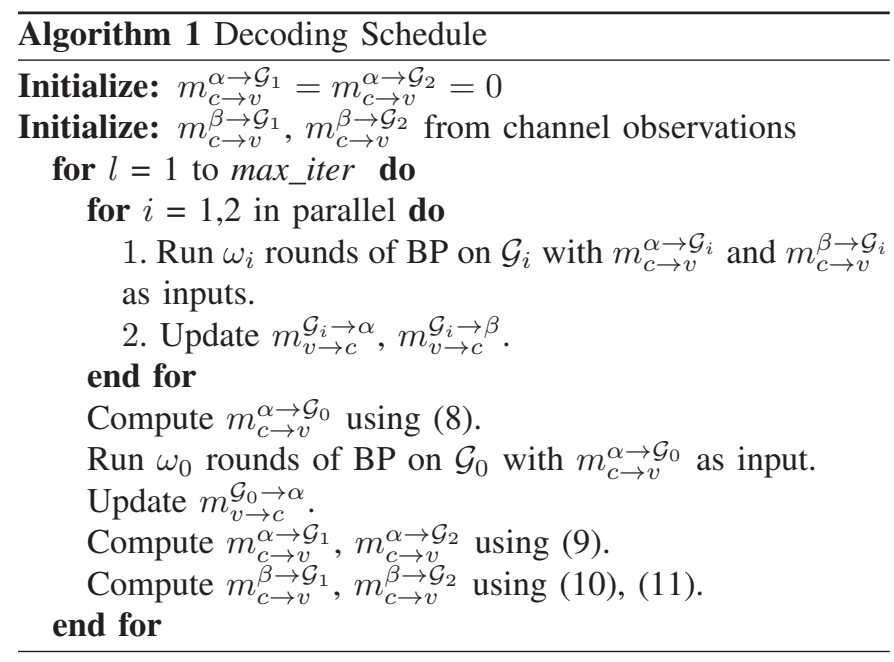

After a fixed number of global iterations, a hard decision is taken on the value of the source bits $\mathbf{x}_{s}$, based on the sign of the corresponding messages at the variable nodes which is obtained by adding the messages from all incident edges. Note that as in the original QMF strategy, no hard decisions are made on the relay transmissions.

\section{Non-binary signaling}

To extend our scheme to higher order constellations, we adopt a 2-step procedure-namely coding in binary, followed by modulating the coded bits to the transmit constellation. The (non-binary) code and membership functions then factorize as

$$
\begin{aligned}
\mathbb{1}_{\left\{\mathbf{x}_{\mathbf{s}} \in \mathcal{C}\right\}} & =\mathbb{1}_{\left\{\mathbf{c}_{\mathbf{s}} \in \mathcal{C}^{\prime}\right\}} \cdot \mathbb{1}_{\left\{\Psi_{v}^{s}\left(\mathbf{c}_{\mathbf{s}}\right)=\mathbf{x}_{\mathbf{s}}\right\}} \\
\mathbb{1}_{\left\{\left(\mathbf{x}_{\mathbf{Q}} \cup \mathbf{x}_{\mathbf{R}}\right) \in \mathcal{M}\right\}} & =\mathbb{1}_{\left\{\left(\mathbf{x}_{\mathbf{Q}} \cup \mathbf{c}_{\mathbf{R}}\right) \in \mathcal{M}^{\prime}\right\}} \cdot \mathbb{1}_{\left\{\Psi_{v}^{R}\left(\mathbf{c}_{\mathbf{R}}\right)=\mathbf{x}_{\mathbf{R}}\right\}}
\end{aligned}
$$

where $\mathbf{c}_{\mathbf{s}}$ and $\mathbf{c}_{\mathbf{R}}$ denote the binary coded and mapped vectors at the source and relays respectively, and $\Psi_{v}^{s}$ and $\Psi_{v}^{R}$ are the vector extensions of the constellation maps. $\mathcal{C}^{\prime}$ and $\mathcal{M}^{\prime}$ denote the binary codebooks at the source and the relays.

From the perspective of the decoder, each type $\alpha$ node now has $k_{s}$ connecting edges to $\mathcal{G}_{0}$ and $b$ connecting edges to $\mathcal{G}_{i}$ while each type $\beta$ check node has $k_{R 1}$ and $k_{R 2}$ connecting edges to $\mathcal{G}_{1}$ and $\mathcal{G}_{2}$ respectively, where $k_{s}, k_{R 1}$ and $k_{R 2}$ represent the number of bits-per-symbol of the constellations $\mathcal{A}_{s}, \mathcal{A}_{R 1}$ and $\mathcal{A}_{R 2}$.

In general $k_{s}, k_{R 1}, k_{R 2}$ may be chosen to be different from each other. In our setup, we restrict $k_{R 1}$ and $k_{R 2}$ to be greater than or equal to $k_{s}$. At the $i^{t h}$ relay, if the constellation has $k_{R i}>b$ we use a rate $\frac{b}{k_{R i}}$ LDPC code to encode $\mathbf{x}_{Q}$ to $\mathbf{c}_{R}$. The encoding algorithm is the one in [8], which retains the input word as a subset of the codeword. In this case, the following equivalence holds at the relay: $\mathbb{1}_{\left\{\left(\mathbf{x}_{\mathbf{Q}} \cup \mathbf{c}_{\mathbf{R}}\right) \in \mathcal{M}^{\prime}\right\}} \equiv$ $\mathbb{1}_{\left\{\mathbf{c}_{\mathbf{R}} \in \mathcal{M}^{\prime}\right\}}$.

\section{Numerical EVAluations}

For the QMF simulations, the codebook $\mathcal{C}^{\prime}$ at the source is chosen from the ensemble for the AWGN channel [9] with maximum degree of variable nodes set to 8 . The mapping codebooks at the relays, $\mathcal{M}_{1}^{\prime}$ and $\mathcal{M}_{2}^{\prime}$ are samples from a $(1,2)$-regular LDPC ensemble ${ }^{2}$. For signaling, we consider QAM constellations with gray-code labeling. Results are presented with 50 global iterations, although 10-20 were mostly sufficient. Within iterations, 10 rounds of BP were performed.

We note that the network is parameterized by the 4 Signalto-Noise-Ratios (SNRs) $\mathcal{S}_{i}=\mathbb{E}\left\{\left|h_{i}\right|^{2}\right\} \cdot \mathcal{P} \quad \forall i \in\{1,2,3,4\}$.

To quantify the performance of our QMF design, we examine three aspects of the system: (i) Error performance with fading links which quantify the cooperative diversity achieved (ii) Rates achieved for a given outage/FER which quantifies spectral efficiency (iii) Examine favorable and unfavorable (in terms of error performance) static configurations.

Cooperative Diversity: Since the individual links are fading, wireless relay networks obtain cooperative diversity by providing multiple transmission paths from source to destination. Fig. 3-4, show that the performance of QMF is within 2-3 $\mathrm{dB}$ of the outage performance of the information-theoretic cutset bound, at moderate SNR. More importantly it performs as well or better than AF-BEAM and OR schemes, which require transmit CSI at the relays. This demonstrates that our implementable designs behave similar to the information-theoretic predictions. Statistically symmetric fading channels seem to be more unfavorable to QMF than statistically asymmetric channels. This is not unexpected since information theory predicts that rate advantages of QMF over other schemes is more pronounced in asymmetric conditions [2].

Spectral efficiency: We saw in Theorem 2.1 that a consequence of the approximate optimality of QMF is that it achieves the optimal multiplexing rate for a given error performance (diversity order). In Fig. 5-6 we explore performance at higher spectral efficiencies through non-binary signaling. We see that our LDPC-based design achieves spectral efficiency within $3 \mathrm{~dB}$ of optimal in asymmetric channels at moderate SNRs, without transmit CSI. We believe that the deterioration in performance for high-rates (3 bits/transmission) in symmetric channels is primarily because we have not optimized the LDPC designs.

Static channels: In Fig. 7-8 we evaluate the performance when the channel amplitudes are static and known but the phases still vary randomly from block-to-block. When the channel amplitudes are identical, QMF performance is relatively the worst. This is because bootstrapping the iterativedecoding becomes the bottleneck due to the symmetric multiple access interference at the last hop. When there is asymmetry, this issue disappears as seen in Fig. 8. Fig. 3-4 demonstrate that in fading channels, such situations occur rarely, and so QMF performs better on the average.

\footnotetext{
${ }^{2}$ Although a $(1,2)$-regular code does not by itself exhibit good performance, we found it to work better as component codes than most ad-hoc choices, including the (3,6)-regular ensemble, repeat-accumulate codes, and also the specially designed ensemble for the MAC channel in [8].
} 


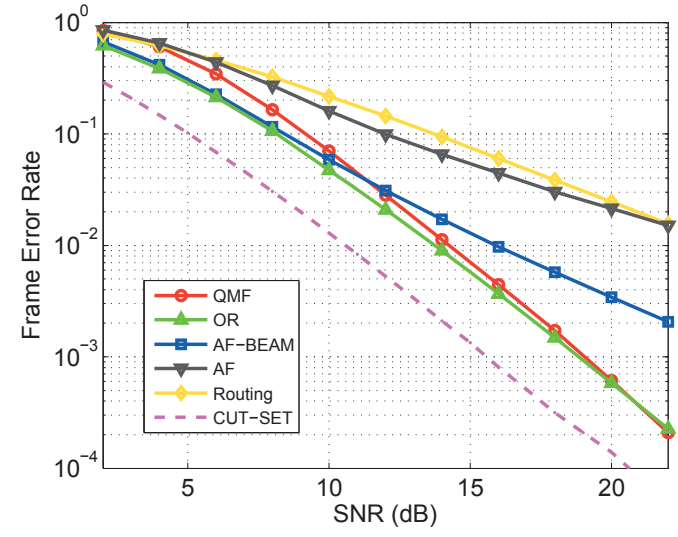

Fig. 3. FER for symmetric block-fading: $h_{1}, h_{2}, h_{3}, h_{4} \sim$ i.i.d $\mathcal{C N}(0,1)$. Rate $=1 \mathrm{bit} / \mathrm{sec} / \mathrm{Hz}$. Modulation: 4-QAM. SNR axis represents $\mathcal{S}_{1}$.

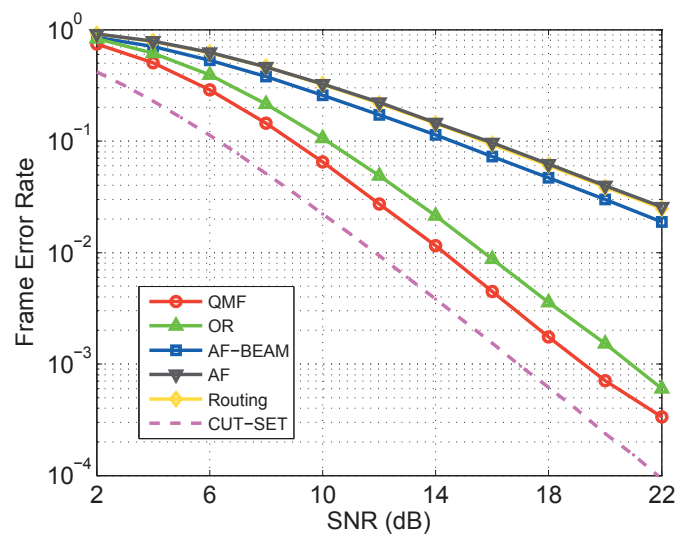

Fig. 4. FER for asymmetric block-fading: $h_{1}, h_{4} \sim$ i.i.d $\mathcal{C N}(0,1) ; h_{2}, h_{3} \sim$ i.i.d $\mathcal{C N}\left(0, \frac{1}{100}\right)$. Rate $=2$ bits $/ \mathrm{sec} / \mathrm{Hz}$. Modulation: 16-QAM. SNR axis represents $\mathcal{S}_{2}$.

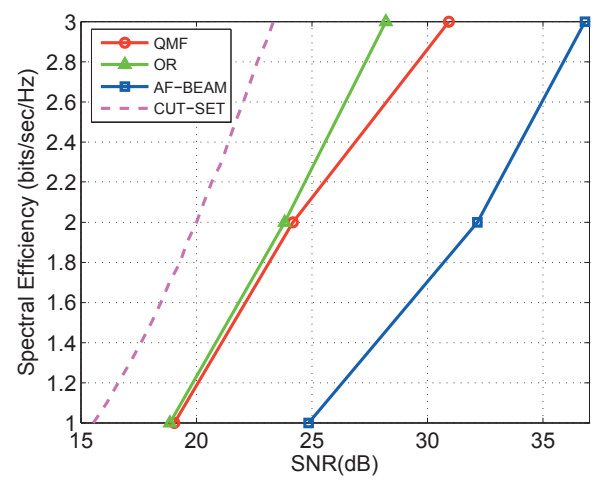

Fig. 5. Spectral Efficiency for symmetric block-fading at $\mathrm{FER}=10^{-3}$. $h_{1}, h_{2}, h_{3}, h_{4} \sim$ i.i.d $\mathcal{C N}(0,1)$. SNR axis represents $\mathcal{S}_{1}$.

\section{REFERENCES}

[1] B. E. Schein, "Distributed Coordination in Network Information Theory", Ph.D thesis, Massachusetts Institute of Technology, Sept. 2001.

[2] S. Avestimehr, S. N. Diggavi and D. Tse, "Wireless network information flow: a deterministic approach", IEEE Trans. on Infor. Theory, vol. 57, no. 4, pp. 1872-1905, Apr. 2011.

[3] S. Avestimehr, S N. Diggavi and D. Tse, "Information flow over compound wireless relay networks", IEEE Zurich Seminar on Communications (IZS), pp. 92, Zurich, Mar. 2008.

[4] S. Pawar, S. Avestimehr and D. Tse, "Diversity-multiplexing tradeoff of half-duplex relay channel", Proc. of the Annual Allerton Conf. on Comm., Control and Computing, Sept. 2008.

[5] V. Nagpal, I-Hsiang Wang, M. Jorgovanovic, D. Tse, B. Nikolic, "Quantize-map-and-forward relaying: coding and system design",Proc.

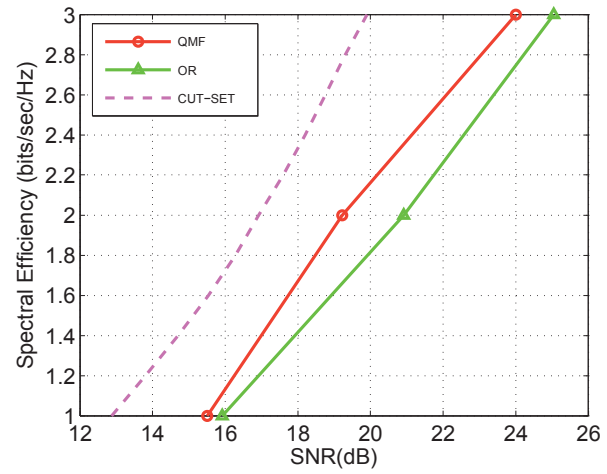

Fig. 6. Spectral Efficiency for asymmetric block-fading at FER $=10^{-3}$. $h_{1}, h_{4} \sim$ i.i.d $\mathcal{C N}(0,1) ; h_{2}, h_{3} \sim$ i.i.d $\mathcal{C N}\left(0, \frac{1}{100}\right)$. SNR axis represents $\mathcal{S}_{2}$.

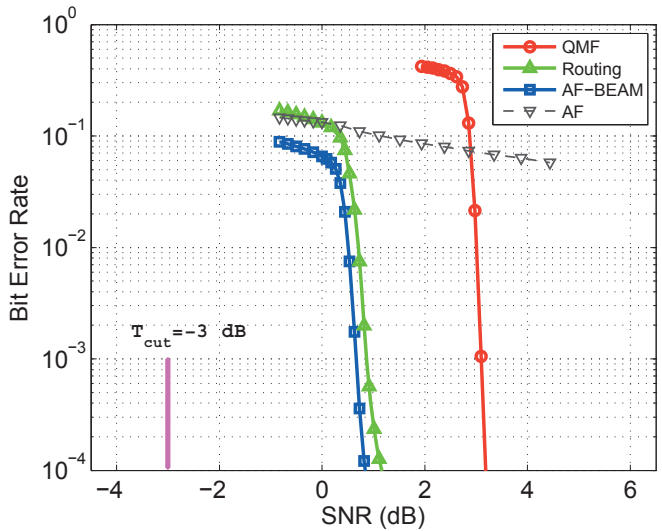

Fig. 7. BER for static channels (symmetric): $\left|h_{1}\right|=\left|h_{2}\right|=\left|h_{3}\right|=\left|h_{4}\right|$. Rate $=1 \mathrm{bit} / \mathrm{sec} / \mathrm{Hz}$. Modulation: 4-QAM. SNR axis represents $\mathcal{S}_{1}$.

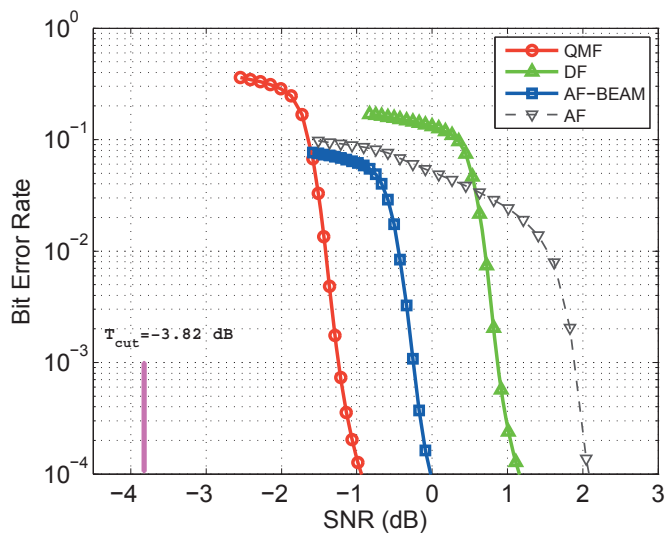

Fig. 8. BER for static channels (asymmetric): $\left|h_{1}\right|=\left|h_{4}\right|=10\left|h_{2}\right|=$ $10\left|h_{3}\right|$. Rate $=1$ bit/sec/Hz. Modulation: 4-QAM. SNR axis represents $\mathcal{S}_{2}$.

of the Annual Allerton Conf. on Comm., Control and Computing, Sept. 2010.

[6] A. Ozgur and S. Diggavi, "Approximately achieving Gaussian relay network capacity with lattice codes", Proc. of IEEE Int. Symp. on Info. Theory (ISIT), 2010.

[7] A. Bletsas, A. Khisti, D. P. Reed, A. Lippman, "A simple cooperative diversity method based on network path selection", IEEE Journal on Selected Areas in Communication, vol. 24, no. 3, pp. 659-672, Mar. 2006.

[8] T. Richardson and R. Urbanke, "Modern Coding Theory", Cambridge University Press, 2008

[9] T. Richardson, M. A. Shokrollahi, R. Urbanke, "Design of capacityapproaching irregular low-density parity-check codes", IEEE Trans. Inform. Theory, vol. 47, pp. 619-637, 2001. 